\title{
On the Field Focusing Effect at the Tungsten Fuzzy Nanostructures Formed by Helium Plasmas
}

\author{
Katsuaki TANABE \\ Department of Chemical Engineering, Kyoto University, Nishikyo, Kyoto 615-8510, Japan
}

(Received 10 September 2021 / Accepted 12 November 2021)

\begin{abstract}
Tungsten fuzzy nanostructures commonly form on the plasma-facing walls of magnetic-confinement nuclear fusion reactors, induced by the helium plasma irradiation. We calculate the field enhancement factors at the fuzz tips of tungsten, molybdenum, and tantalum, quantitatively representing the degree of field focusing, based on the classical electromagnetic field theory under the quasistatic approximation, for a model system comprising a subwavelength-scale prolate metal hemispheroid protruding from a conducting plane. Field enhancement factors of $2.4 \times 10^{3}, 5.4 \times 10^{6}$, and $2.3 \times 10^{10}$ for the spheroidal aspect ratio of 10,100 , and 1000 , respectively, are observed in the gigahertz regime for the incident electric field parallel to the fuzz, i.e., normal to the reactor wall. Such a potential large field focusing effect may be worth accounting for in the designing and operation of fusion reactors.
\end{abstract}

(C) 2021 The Japan Society of Plasma Science and Nuclear Fusion Research

Keywords: nuclear fusion reactor, plasma-facing wall, electromagnetic field, field enhancement, metal, nanoparticle, fuzzy nanostructure

DOI: $10.1585 / \mathrm{pfr} .16 .1405107$

\section{Introduction}

Tungsten has been the leading candidate material for the plasma-facing walls of nuclear fusion reactors, because of its high melting point, low tritium retention, low sputtering yield, and high thermal conductivity [1-3]. In the prototype reactors of magnetic-confinement fusion, fuzzy nanostructures of tungsten induced by helium plasma irradiation have commonly been observed on the plasmafacing walls after a series of test plasma operation runs, and the mechanism of the fuzz formation has been extensively studied [3-8]. It is known that metal surfaces with sharp curvatures allow electromagnetic fields to concentrate in their vicinity, as known as the working principle of radio antennae and lightning rods. Because the tungsten fuzzes are structurally observed to be highly sharp, there is a concern of field focusing at the fuzz tips, which may potentially cause an undesirable influence on the confined plasmas in fusion reactors. In the present study, we numerically investigate the field focusing effect around fuzzy nanostructures of candidate metals for the plasma-facing wall material, tungsten, molybdenum, and tantalum.

\section{Theory and Calculation Methods}

No focusing of the magnetic field occurs on the tungsten nanostructures because tungsten is a non-magnetic material, i.e., its relative permeability is unity and equal to that of the vacuum space. We therefore only investigate the focusing effect of the electric field. Firstly, let us consider the electric field profile around a spherical metal

author'se-mail: tanabe@cheme.kyoto-u.ac.jp object in vacuum and the field enhancement factor, defined as the ratio of the field intensity around the object to that in the absence of the object, or the original incident field [9]. Because the scale of the subject tungsten nanostructures is significantly smaller than the wavelength of the electromagnetic field to be applied in the nuclear fusion reactors, the electrostatic approximation can be employed for the calculations, where the phase retardation is negligible throughout the object [10]. Consider a homogeneous, isotropic sphere placed in a medium in which there exists a uniform static electric field $\vec{E}_{0}=E_{0} \hat{e}_{x}$. If the permittivities or dielectric constants of the sphere and the surrounding medium are different, a charge will be induced on the surface of the sphere. The originally uniform field will be therefore distorted by the introduction of the sphere. Based on the classical electromagnetic field theory, the electric fields inside and outside the sphere, $\vec{E}_{1}$ and $\vec{E}_{2}$, respectively, are derivable from scalar potentials $\Phi_{1}(r, \theta)$ and $\Phi_{2}(r, \theta)$ :

$$
\vec{E}_{1}=-\nabla \Phi_{1}, \vec{E}_{2}=-\nabla \Phi_{2}
$$

where

$$
\nabla^{2} \Phi_{1}=0(r<a), \nabla^{2} \Phi_{2}=0(r>a)
$$

where $r$ is the radial distance of the observing point from the center of the sphere, $\theta$ is the polar angle of the observing point with respect to the $x$-axis or the direction of $\vec{E}_{0}$, and $a$ is the radius of the sphere. Because of the symmetry of the system, the potentials are independent of the azimuthal angle $\varphi$. At the boundary between the sphere and 
the medium, the potentials must satisfy:

$$
\Phi_{1}=\Phi_{2}, \varepsilon_{1} \frac{\partial \Phi_{1}}{\partial r}=\varepsilon_{m} \frac{\partial \Phi_{2}}{\partial r}(r=a),
$$

where $\varepsilon_{1}$ and $\varepsilon_{m}$ are the frequency-dependent complex permittivities or dielectric functions of the sphere and the surrounding medium, respectively. It is additionally required that

$$
\lim _{r \rightarrow \infty} \Phi_{2}=-E_{0} r \cos \theta=-E_{0} x,
$$

that is, the electric field far from the sphere is the unperturbed original field. It can be derived that the potentials

$$
\begin{aligned}
& \Phi_{1}=-\frac{3 \varepsilon_{m}}{\varepsilon_{1}+2 \varepsilon_{m}} E_{0} r \cos \theta, \\
& \Phi_{2}=-E_{0} r \cos \theta+a^{3} E_{0} \frac{\varepsilon_{1}-\varepsilon_{m}}{\varepsilon_{1}+2 \varepsilon_{m}} \frac{\cos \theta}{r^{2}},
\end{aligned}
$$

satisfy Eqs. (2) - (4). These solutions for the potentials could also be derived rigorously by using Legendre polynomials. From Eqs. (1) and (6), the electric field outside the sphere can be expressed as:

$$
\begin{aligned}
\vec{E}_{2}=E_{0}\{ & \left(1+2 \frac{a^{3}}{r^{3}} \frac{\varepsilon_{1}-\varepsilon_{m}}{\varepsilon_{1}+2 \varepsilon_{m}}\right) \hat{e}_{r} \cos \theta \\
& \left.+\left(-1+\frac{a^{3}}{r^{3}} \frac{\varepsilon_{1}-\varepsilon_{m}}{\varepsilon_{1}+2 \varepsilon_{m}}\right) \hat{e}_{\theta} \sin \theta\right\},
\end{aligned}
$$

where $\hat{e}_{r}$ and $\hat{e}_{\theta}$ are the unit vectors to $r$ and $\theta$ directions, respectively, and thus:

$$
\begin{aligned}
\left|\vec{E}_{2}\right|^{2}=\left|\vec{E}_{0}\right|^{2} & \left(\left|1+2 \frac{a^{3}}{r^{3}} \frac{\varepsilon_{1}-\varepsilon_{m}}{\varepsilon_{1}+2 \varepsilon_{m}}\right|^{2} \cos ^{2} \theta\right. \\
& \left.+\left|-1+\frac{a^{3}}{r^{3}} \frac{\varepsilon_{1}-\varepsilon_{m}}{\varepsilon_{1}+2 \varepsilon_{m}}\right|^{2} \sin ^{2} \theta\right) .
\end{aligned}
$$

The electric field intensity will be therefore maximized at the direction $\theta=0, \pi$ for most cases, and the field enhancement factor is defined as:

$$
\eta \equiv \frac{\left|\vec{E}_{2}\right|^{2}}{\left|\vec{E}_{0}\right|^{2}}=\left|1+2 \frac{a^{3}}{r^{3}} \frac{\varepsilon_{1}-\varepsilon_{m}}{\varepsilon_{1}+2 \varepsilon_{m}}\right|^{2} .
$$

Note that $\eta$ is defined as the ratio of field intensities and not field magnitudes. As found in Eq. (9), the largest field enhancement or focusing occurs immediately adjacent to the surface of the sphere, $r=a$, and therefore we calculate $\eta$ for the point $r=a$ :

$$
\eta=\left|1+2 \frac{\varepsilon_{1}-\varepsilon_{m}}{\varepsilon_{1}+2 \varepsilon_{m}}\right|^{2}=\left|\frac{3 \varepsilon_{1}}{\varepsilon_{1}+2 \varepsilon_{m}}\right|^{2} .
$$

It should be noted that the field enhancement factor $\eta$, for our formulation, is thus independent of the size of the object. For a prolate spheroid, $\eta$ becomes maximum at its tips and for the alignment of its major axis parallel to $\vec{E}_{0}$, and is formulated as $[10,11]$ :

$$
\eta=\left|\frac{\varepsilon_{1}}{\varepsilon_{m}+L_{1}\left(\varepsilon_{1}-\varepsilon_{m}\right)}\right|^{2},
$$

where $L_{1}$ is the geometrical factor for the major axis of the prolate spheroid, calculated as:

$$
L_{1}=\frac{1-e^{2}}{e^{2}}\left(-1+\frac{1}{2 e} \ln \frac{1+e}{1-e}\right) .
$$

$e$ is the eccentricity of the spheroidal shape:

$$
e=\sqrt{1-\frac{b^{2}}{a^{2}}}
$$

where $a$ and $b$ are the radii of the major axis and the minor axes of the prolate spheroid, respectively. $L_{1}$ of $1 / 3$ corresponds to the case of a sphere, Eq. (10).

So far, we have discussed an isolated object suspended in a medium. Let us move onto a more practical model system to mimic the tungsten fuzz on the plasma-facing wall. We consider a prolate hemispheroid normally protruding from a perfectly conducting flat plane with an infinite area, as conceptually depicted in Fig. 1. The presence of the perfectly conducting plane causes the component of the incident electric field normal to the surface to be doubled, while canceling the tangential part $[12,13]$. Therefore, the change of the model system from the isolated prolate spheroid to the prolate hemispheroid protruding from the conducting plane simply doubles $\vec{E}_{2}$, due to the doubled $\vec{E}_{0}$ in the absence of the hemispheroid but in the presence of the conducting plane relative to the primitive $\vec{E}_{0}$ in the total absence of the hemispheroid and the plane. Nevertheless, we should redefine $\eta$ to represent the influence of the formation of the tungsten fuzz on the plasmafacing wall, as the ratio of the intensity of $\vec{E}_{2}$ to that of $\vec{E}_{0}$ in the absence of the hemispheroid but in the presence

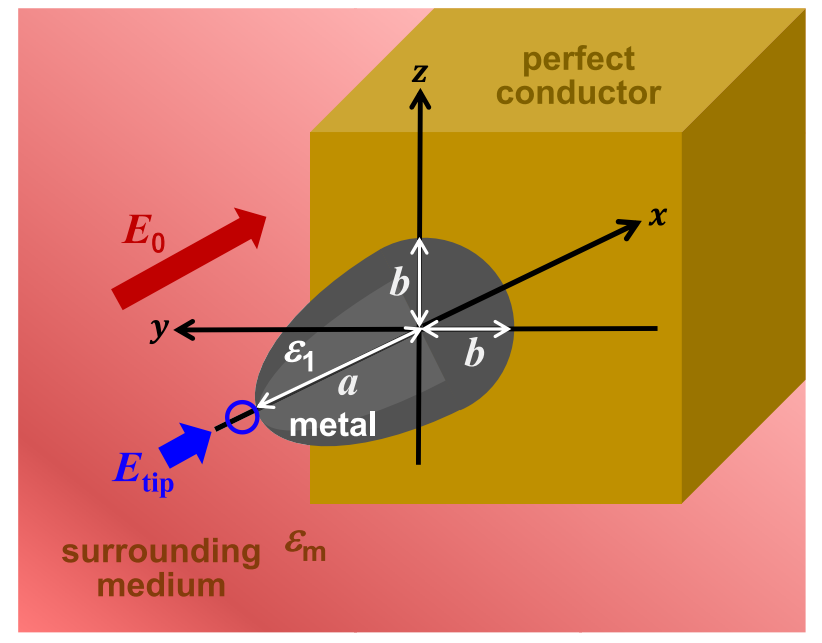

Fig. 1 Conceptual schematic of the model system for the metal fuzz on the reactor wall considered for the calculations in this study. A prolate metal hemispheroid normally protrudes from a perfectly conducting flat plane with an infinite area, and a uniform static electric field primitively presents in the direction parallel to the major axis of the hemispheroid. 
of the conducting plane. As the result, $\eta$ of our interest for the model system, comprising a prolate metal hemispheroid normally protruding from a perfectly conducting flat plane, is eventually equal to that in Eq. (11). We thus calculate the field enhancement factor $\eta$ at the tip of the prolate hemispheroid representing the degree of field focusing at the tungsten fuzz. Along with tungsten, other candidate metals with high melting temperatures for the plasma-facing walls, molybdenum and tantalum $[3,5,6,8]$, are also investigated. As references, we also calculate $\eta$ for prolate hemispheroids of noble metals, gold and silver, as the representatives of highly electrically active materials represented by their highest conductivities among metals. For $\varepsilon_{1}$, the empirical complex dielectric constants of tungsten, molybdenum, and tantalum on frequency reported in Ref. [14] are employed. Those of gold and silver listed as polynomial functions of freespace wavelength in Refs. [9] and [15], originally fit to the data of Ref. [14], are used for higher and lower frequencies, respectively. We assume that $\varepsilon_{m}=1$ throughout the entire frequencies, representing vacuum or any dilute gas as the surrounding medium.

\section{Results and Discussion}

Figure 2 presents the calculated field enhancement factors $\eta$ in dependence on frequency at the tips of prolate hemispheroids of tungsten, molybdenum, tantalum, gold, and silver normally protruding from a perfectly conducting flat plane with an infinite area. These calculations of $\eta$, based on the classical electromagnetic field theory in the quasistatic limit, quantitatively represent how much energy is concentrated from the incident electric power, and thus the degree of field focusing. Figures 2 (a), (b), and (c) plot the calculation results of $\eta$ for the conditions of the aspect ratio of the prolate hemispheroids, $a / b$, of 1,10 , and 100, respectively. In general, as the curvature of the spheroidal tip becomes sharper, which corresponds to the increase of the aspect ratio, the field enhancement effect becomes larger. Note that, for the case that the aspect ratio is 1 , we practically input $b / a=0.999999$ to circumvent diverging numerical errors in the computation of Eq. (12). Figure 2 (a), corresponding to the case of hemispheres, the candidate metals for plasma-facing walls, tungsten, molybdenum, and tantalum, exhibit small field enhancement factors of about 10 throughout the entire frequencies. Gold and silver hemispheres show larger $\eta$ of about 20 and 100 , respectively, presumably due to their high electrical conductivities that cause small damping loss. The resonances observed for the gold and silver hemispheres at about 600 and $800 \mathrm{THz}$, corresponding to the freespace wavelengths of about 550 and $400 \mathrm{~nm}$, respectively, are well known for their spherical nanoparticles. Figure 2 (b), the results for prolate metal hemispheroids with an aspect ratio of 10 , presents large $\eta$ in the range of 100 - 10000 for tungsten, molybdenum, and tantalum. The resonant frequencies are found to be $150 \mathrm{THz}$ or $2.0 \mu \mathrm{m}\left(\eta=2.0 \times 10^{4}\right)$, (a) Aspect ratio $=1$

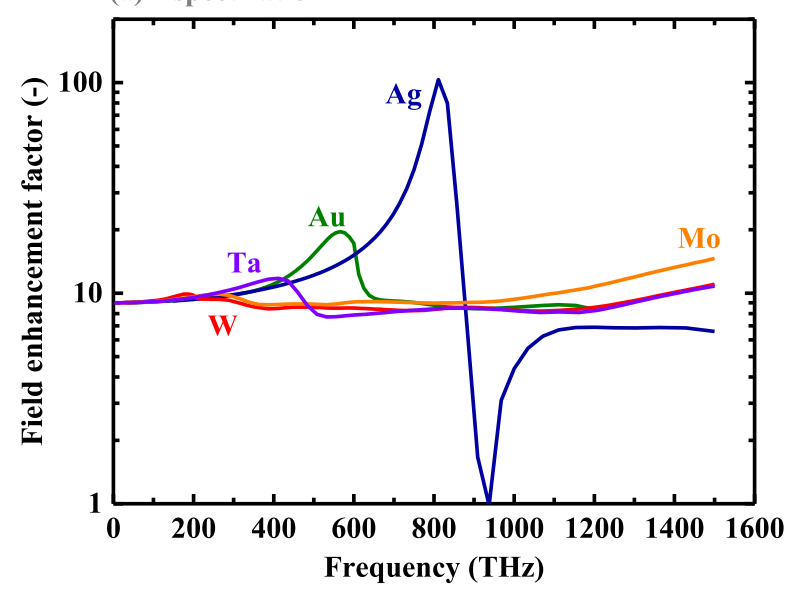

(b) Aspect ratio $=10$

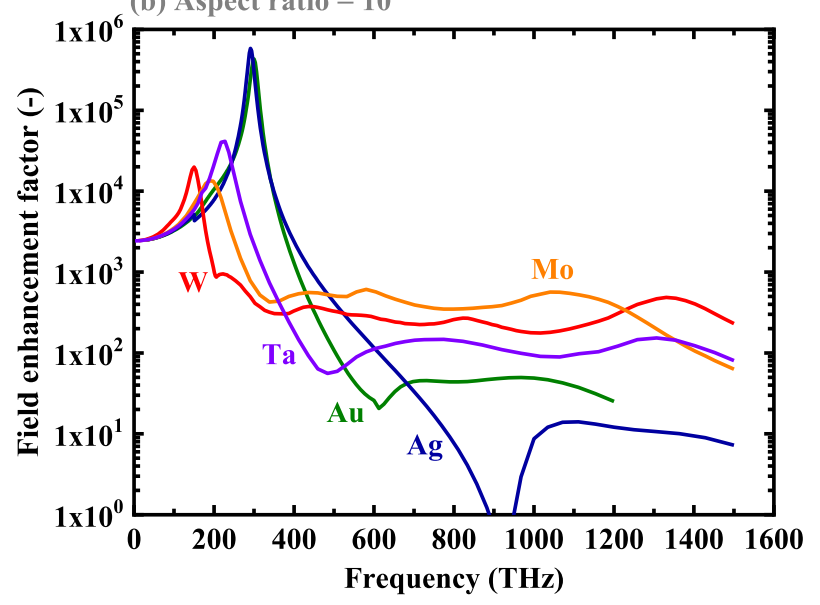

(c) Aspect ratio $=100$

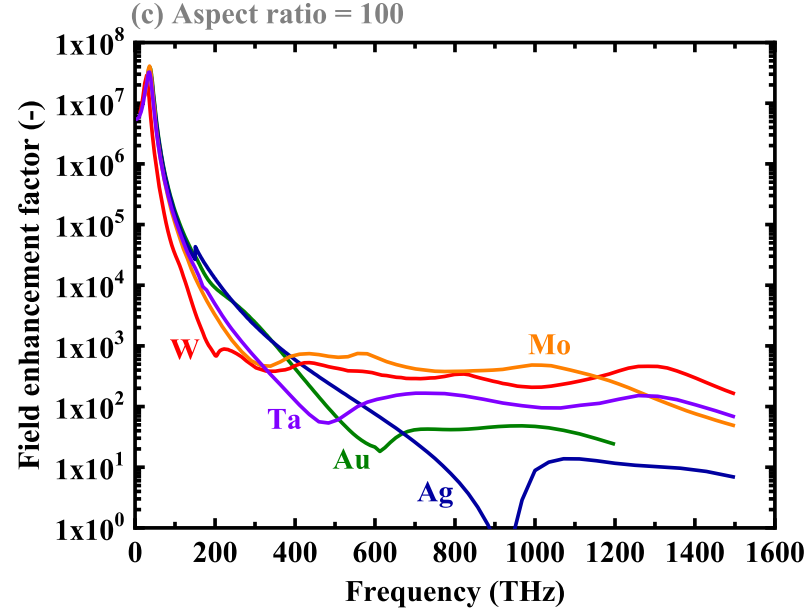

Fig. 2 Calculated field enhancement factor $\eta$ in dependence on frequency at the tips of prolate hemispheroids of tungsten, molybdenum, tantalum, gold, and silver, with aspect ratio $a / b$ of (a) 1 , (b) 10 , and (c) 100 .

$190 \mathrm{THz}$ or $1.6 \mu \mathrm{m}\left(\eta=1.3 \times 10^{4}\right), 220 \mathrm{THz}$ or $1.4 \mu \mathrm{m}$ $\left(\eta=4.1 \times 10^{4}\right)$ for tungsten, molybdenum, and tantalum, respectively. Again, gold and silver exhibit even larger $\eta$, close to a million for the peaks, on their resonance at about $300 \mathrm{THz}(1 \mu \mathrm{m})$. Nevertheless, for such a frequency region of hundreds of terahertzes, the accuracy of our cal- 
culation with the electrostatic approximation is debatable because the lengths of the fuzzes observed in the experiments are generally about $1 \mu \mathrm{m}$ or larger, and thus comparable to the wavelengths. However, it is worth mentioning that the plasma-heating frequencies used in fusion reactors are typically below $1 \mathrm{THz}$ [16], for which the electrostatic approximation is valid. In this sense, we should focus on the lower frequency regions of the calculated $\eta$ spectra. For the sharp prolate hemispheroids, resembling the tungsten and molybdenum fuzzy nanostructures experimentally observed [3-6, 8], with an aspect ratio of 100, very large $\eta$ over ten million are calculated on the resonance (at 30 $40 \mathrm{THz}$, or $8-10 \mu \mathrm{m}$, for all the metals) in Fig. 2 (c). Thus, through Figs. 2 (a) - (c), it is observed that $\eta$ significantly increases with the aspect ratio of the prolate hemispheroid, or the sharpness of the metal tip. Large-aspect-ratio metal structures or high-curvature edges of surface irregularities exhibit high polarizabilities and, thus, large dipole moments, particularly at the resonance, to produce strong local field enhancement in the vicinity of such edges. Even at the lower frequency limit in Fig. 2 (c), representing the typical plasma-heating frequencies used in fusion reactors, $\eta$ is observed larger than a million, and therefore it may be important to account for the field focusing effect particularly in the presence of sharp fuzzy metal nanostructures during the nuclear fusion reactor operation. Through Figs. 2 (a) - (c), it is observed that in the lower frequency limit, $\eta$ converges to a certain value common for all of the metal elements for each aspect ratio of the prolate metal spheroid. This $\eta$ converging trend is because the modulus of the real and imaginary parts of dielectric functions of metals, $\varepsilon_{1}$, becomes incomparably larger than those of the surrounding media such as vacuum and dilute gases, $\varepsilon_{m}$, for lower frequencies, and therefore $\eta$ converges to $1 / L_{1}^{2}$, as understood with Eq. (11). Namely,

$$
\eta=\frac{1}{L_{1}^{2}} \text { (lower frequency limit), }
$$

which is a simple function only of $a / b$, the aspect ratio of the prolate hemispheroid, as understood with Eqs. (12) and (13). In this context, we plot in Fig. 3 the field enhancement factors, $\eta$, in the lower frequency limit, which corresponds to the situation of the typical fields present in the nuclear fusion reactors, in dependence on the aspect ratio, $a / b$, of the prolate metal spheroids protruding from the conducting plane. Importantly, this plot is common for any kind of metal, owing to the principle explained above. It is observed that the convergent $\eta$, again, increases with the aspect ratio of the metal hemispheroids, becoming $2.4 \times 10^{3}, 5.4 \times 10^{6}$, and $2.3 \times 10^{10}$ for the aspect ratio of 10,100 , and 1000, respectively. A series of calculation results delivered in this article indicates that such significantly large field focusing possibly occurs depending on the condition of the present field and the metal fuzzes on the plasma-facing wall, which may be worth being accounted for in the designing and operation of fusion reac-

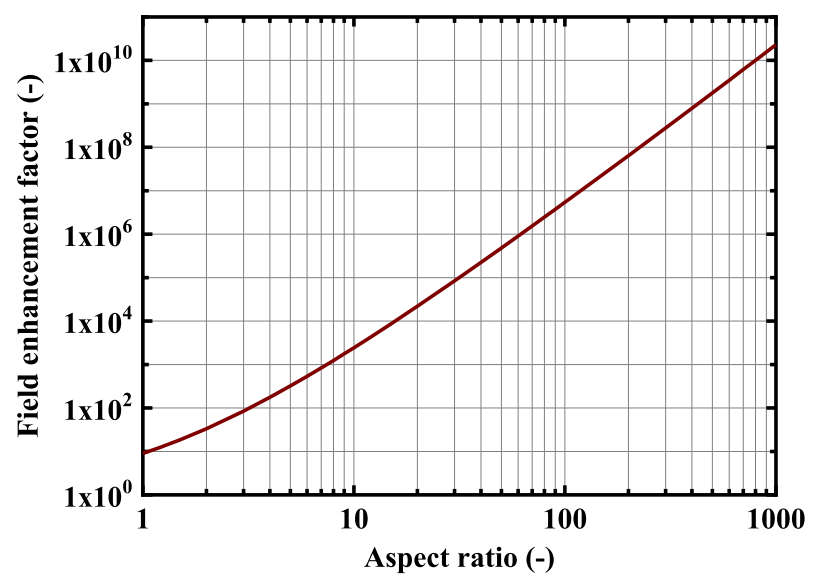

Fig. 3 Field enhancement factors $\eta$ in the lower frequency limit, common for all metal elements, in dependence on spheroidal aspect ratio $a / b$.

tors. In the present study, we calculated field enhancement on single fuzzes for simplicity, but forests of fuzzes are observed in the experiments [3-6,8]. For the case that the separation of the fuzzes is comparable to the wavelength, the interference effect has to be accounted for. We are going to carry out calculations for such ensembles of fuzzes in our future study.

\section{Conclusions}

In this study, we numerically investigated the field focusing effect on fuzzy metal nanostructures formed on the plasma-facing walls of magnetic-confinement nuclear fusion reactors. Specifically, we calculated the field enhancement factors at the fuzz tips of candidate metals for the plasma-facing wall material, tungsten, molybdenum, and tantalum. We modelled the fuzz on the reactor wall as a prolate metal hemispheroid normally protruding from a perfectly conducting flat plane with an infinite area. For the calculations based on the classical electromagnetic field theory, we employed the quasistatic approximation, accounting for the scale of the experimentally observed fuzzy metal structures significantly smaller than the typical applied field wavelengths in plasma fusion reactors. For the incident electric field parallel to the fuzz (i.e., normal to the reactor wall), our calculations observed large field enhancement factors of $2.4 \times 10^{3}, 5.4 \times 10^{6}$, and $2.3 \times 10^{10}$ for the spheroidal aspect ratio of 10,100 , and 1000, respectively, for frequencies below the terahertz regime.

This study was supported in part by the Japan Society for the Promotion of Science (JSPS).

[1] J. Davis, V. Barabash, A. Makhankov, L. Plöchl and K. Slattery, J. Nucl. Mater. 258-263, 308 (1998).

[2] B. Lipschultz, Nucl. Fusion 47, 1189 (2007).

[3] G. Sinclair, J.K. Tripathi, P.K. Diwakar and A. Hassanein, Nucl. Fusion 56, 036005 (2016). 
[4] A.M. Ito, A. Takayama, Y. Oda, T. Tamura, R. Kobayashi, T. Hattori, S. Ogata, N. Ohno, S. Kajita, M. Yajima, Y. Noiri, Y. Yoshimoto, S. Saito, S. Takamura, T. Murashima, M. Miyamoto and H. Nakamura, Nucl. Fusion 55, 073013 (2015).

[5] P. Fiflis, N. Connolly and D.N. Ruzic, J. Nucl. Mater. 482, 201 (2016).

[6] K.D. Hammond, Mater. Res. Express 4, 104002 (2017).

[7] A.M. Ito, A. Takayama and H. Nakamura, Plasma Fusion Res. 13, 3403061 (2018).

[8] S. Kajita, S. Kawaguchi, N. Ohno and N. Yoshida, Sci. Rep. 8, 56 (2018).
[9] K. Tanabe, J. Phys. Chem. C 112, 15721 (2008).

[10] C.F. Bohren and D.R. Huffman, Absorption and Scattering of Light by Small Particles (Wiley, Weinheim, 1983).

[11] P.F. Liao and A. Wokaun, J. Chem. Phys. 76, 751 (1982).

[12] J. Gersten and A. Nitzan, J. Chem. Phys. 73, 3023 (1980).

[13] C.K. Chen, T.F. Heinz, D. Ricard and Y.R. Shen, Phys. Rev. B 27, 1965 (1983).

[14] E.D. Palik, Handbook of Optical Constants of Solids (Academic Press, Orlando, 1985).

[15] N. Fukuoka and K. Tanabe, J. Appl. Phys. 126, 023102 (2019).

[16] H. Kasahara, J. Plasma Fusion Res. 96, 651 (2020). 